\title{
Secrecy Performance of Mobile Image Transmission Networks
}

\author{
Lingwei $\mathrm{Xu}{ }^{1}, \mathrm{Xu} \mathrm{Yu}{ }^{1}$, Han Wang ${ }^{2}$, Xinjie Wang ${ }^{3}$ and Jingjing Wang ${ }^{1}$ \\ \{gaomilaojia2009@163.com ${ }^{1}$,yuxu0532@163.com ${ }^{2}$, hanwang1214@126.com ${ }^{3}$ \} \\ 1.Department of Information Science and Technology, Qingdao University of Science and Technology, \\ Qingdao 266061, China; 2.College of Physical Science and Engineering, Yichun University, Yichun \\ 336000, China;3.College of Information and Control Engineering, Qingdao University of Technology, \\ Qingdao 266520, China
}

\begin{abstract}
Due to the user mobility, the physical layer security of mobile image transmission networks is an important challenging task.In this paper, based on the classic Wyner wiretap model, the secrecy performance of mobile image transmission networks is investigated. We derive exact closed-form expressions for the average secrecy capacity (ASC). By Monte-Carlo simulations, we verify the accuracy of the derived theoretical results.
\end{abstract}

Keywords: mobile image transmission networks; physical layer security; average secrecy capacity

\section{Introduction}

In recent years, based on user quality of experience (QoE), the mobile image transmission has attracted wide research interest [1,2]. In [3], the authors used Genetic Algorithm (GA) and Particle Swarm Optimization (PSO) techniques to improve the performance of the image steganography system.

Due to the user mobility, the physical layer security of wireless communications is an important challenging task. [4] designed a CP based secure wireless video data transmission. In [5], the security and privacy issues of multimedia services were investigated.Based on artificial-noise-aided beamforming, an optimized secure multi-antenna transmission approach was presented in [6]. [7] designed a multiple-input single-output downlink system to keep the message secret to the energy-harvesting receivers while maximizing the information rate at the information receiver.

However, in [4-7], the physical layer security performance is only considered for Rayleigh and Nakagami-m fading channels. Rayleigh and Nakagami-m fading channels are not fit to model the mobile image transmission[8]. To the best of our knowledge, the physical layer security of mobile image transmission networks has not been considered in the literature. In this paper,we use the average secrecy capacity (ASC) performance to measure the quality of mobile image transmission networks over 2-Nakagami fading channels. When we obtain the poor ASC performance, it means that the mobile image transmission is interrupted. We derive closed-form expressions for the ASC. By Monte-Carlo simulations, we verify the accuracy of the derived theoretical results.

The rest of the paper is organized as follows. The mobile Wyner wiretap model is presented in Section 2.The closed-form ASC expressions are derived in Section 3.Monte- 
Carlo simulation results are provided in Section 4. Finally, some concluding remarks are given in Section 5.

\section{The System Model}

Figure 1. shows the mobile Wyner wiretap model. There is a mobile source (S), a mobile eavesdropper (E), and a mobile destination (D).They are all equipped with a single antenna.

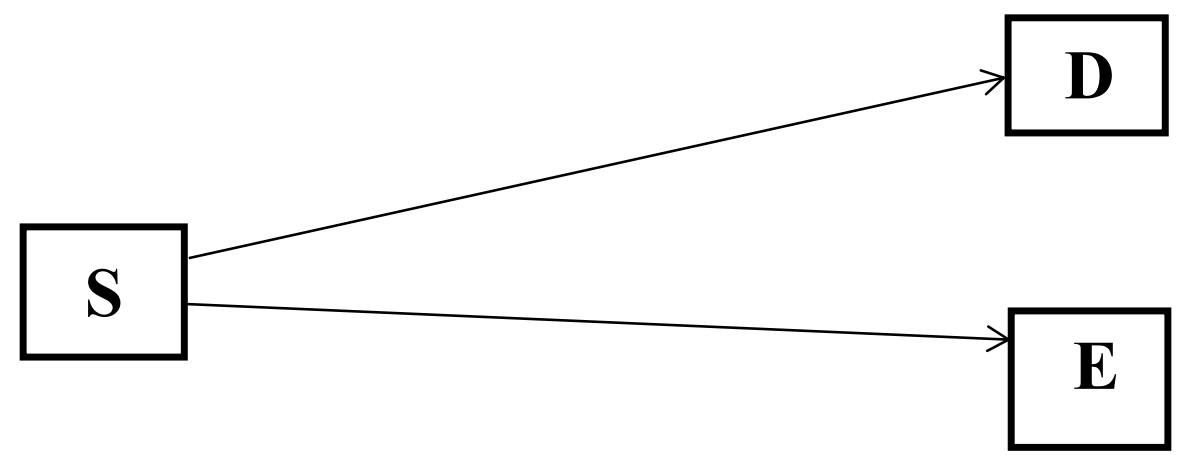

Fig. 1. The system model

We use $h=h_{k}, \mathrm{k} \in\{\mathrm{D}, \mathrm{E}\}$, to represent the complex channel coefficients.To represent the locations of the $\mathrm{D}$ and $\mathrm{E}$ relative to the $\mathrm{S}$, the relative geometrical gain of the $\mathrm{S} \rightarrow \mathrm{D}$ channel is $G_{\mathrm{D}}$, and the relative geometrical gain of the $\mathrm{S} \rightarrow \mathrm{E}$ channel is $G_{\mathrm{E}}[9]$.

$\mathrm{S}$ transmits the image signal $x, \mathrm{D}$ and $\mathrm{E}$ receive the signals as

$$
\begin{aligned}
& r_{\mathrm{D}}=\sqrt{G_{\mathrm{D}} E} h_{\mathrm{D}} x+n_{\mathrm{D}} \\
& r_{\mathrm{E}}=\sqrt{G_{\mathrm{E}} E} h_{\mathrm{E}} x+n_{\mathrm{E}}
\end{aligned}
$$

where $E$ is the energy used by S, the mean and variance of $n_{\mathrm{D}}$ and $n_{\mathrm{E}}$ are 0 and $N_{0} / 2$.

$\mathrm{D}$ receives the signal-to-noise ratio $(\mathrm{SNR})$ as

$$
\begin{gathered}
\gamma_{\mathrm{D}}=K G_{\mathrm{D}}\left|h_{\mathrm{D}}\right|^{2} \bar{\gamma} \\
\bar{\gamma}=\frac{E}{N_{0}} \\
\overline{\gamma_{\mathrm{D}}}=K G_{\mathrm{D}} \bar{\gamma}
\end{gathered}
$$

where $K$ is the relative SNR gain.

E receives the SNR as

$$
\begin{gathered}
\gamma_{\mathrm{E}}=G_{\mathrm{E}}\left|h_{\mathrm{E}}\right|^{2-} \bar{\gamma} \\
\overline{\gamma_{\mathrm{E}}}=G_{\mathrm{E}} \bar{\gamma}
\end{gathered}
$$

The cumulative distribution function of $\gamma_{k}$ is then 


$$
F_{\gamma_{k}}(r)=\frac{1}{\prod_{i=1}^{2} \Gamma\left(m_{i}\right)} G_{1,3}^{2,1}\left[\left.\frac{r}{\overline{\gamma_{k}}} \prod_{i=1}^{2} \frac{m_{i}}{\Omega_{i}}\right|_{m_{1}, m_{2}, 0} ^{1}\right]
$$

where $\Gamma(\cdot)$ is the Gamma function, $m$ is the fading coefficient, and $\Omega$ is a scaling factor.

The corresponding probability density function is given as

$$
f_{\gamma_{k}}(r)=\frac{1}{r \prod_{i=1}^{2} \Gamma\left(m_{i}\right)} G_{0,2}^{2,0}\left[\left.\frac{r}{\overline{\gamma_{k}}} \prod_{i=1}^{2} \frac{m_{i}}{\Omega_{i}}\right|_{m_{1}, \ldots, m_{N}} ^{-}\right]
$$

\section{Average Secrecy Capacity}

[10] gives the instantaneous secrecy capacity as

$$
C_{\mathrm{S}}=\max \left\{\ln \left(1+\gamma_{\mathrm{D}}\right)-\ln \left(1+\gamma_{\mathrm{E}}\right), 0\right\}
$$

So the ASC is given as

$$
\begin{aligned}
\overline{C_{\mathrm{S}}} & =\int_{0}^{\infty} \int_{0}^{\infty} C_{\mathrm{S}}\left(\gamma_{\mathrm{D}}, \gamma_{\mathrm{E}}\right) f\left(\gamma_{\mathrm{D}}, \gamma_{\mathrm{E}}\right) d \gamma_{\mathrm{D}} d \gamma_{\mathrm{E}} \\
& =\int_{0}^{\infty} \int_{0}^{\infty} C_{\mathrm{S}}\left(\gamma_{\mathrm{D}}, \gamma_{\mathrm{E}}\right) f\left(\gamma_{\mathrm{D}}, \gamma_{\mathrm{E}}\right) d \gamma_{\mathrm{D}} d \gamma_{\mathrm{E}} \\
& =\int_{0}^{\infty} \ln \left(1+\gamma_{\mathrm{D}}\right) f_{\mathrm{D}}\left(\gamma_{\mathrm{D}}\right) F_{\mathrm{E}}\left(\gamma_{\mathrm{D}}\right) d \gamma_{\mathrm{D}} \\
& +\int_{0}^{\infty} \ln \left(1+\gamma_{\mathrm{E}}\right) f_{\mathrm{E}}\left(\gamma_{\mathrm{E}}\right) F_{\mathrm{D}}\left(\gamma_{\mathrm{E}}\right) d \gamma_{\mathrm{E}} \\
& -\int_{0}^{\infty} \ln \left(1+\gamma_{\mathrm{E}}\right) f_{\mathrm{E}}\left(\gamma_{\mathrm{E}}\right) d \gamma_{\mathrm{E}} \\
& =\mathrm{M}_{1}+\mathrm{M}_{2}-\mathrm{M}_{3}
\end{aligned}
$$

With the help of [11], $M_{1}$ is given as 


$$
\begin{aligned}
& M_{1}=\frac{1}{\prod_{i=1}^{N} \Gamma\left(m_{i}\right) \prod_{j=1}^{N} \Gamma\left(m_{j}\right)} \times \\
& \int_{0}^{\infty} \ln \left(1+\gamma_{\mathrm{D}}\right) \frac{1}{\gamma_{\mathrm{D}}} G_{0,2}^{2,0}\left[\left.\frac{\gamma_{\mathrm{D}}}{\overline{\gamma_{\mathrm{D}}}} \prod_{i=1}^{2} \frac{m_{i}}{\Omega_{i}}\right|_{m_{1}, m_{2}} ^{-}\right] G_{1,3}^{2,1}\left[\left.\frac{\gamma_{\mathrm{D}}}{\overline{\gamma_{\mathrm{E}}}} \prod_{j=1}^{2} \frac{m_{j}}{\Omega_{j}}\right|_{m_{1}, m_{2}, 0} ^{1}\right] d \gamma_{\mathrm{D}} \\
& =\frac{1}{\prod_{i=1}^{2} \Gamma\left(m_{i}\right) \prod_{j=1}^{2} \Gamma\left(m_{j}\right)} \times \\
& \int_{0}^{\infty} G_{2,2}^{1,2}\left(\gamma_{\mathrm{D}} \mid \begin{array}{l}
1,1 \\
1,0
\end{array}\right) \frac{1}{\gamma_{\mathrm{D}}} G_{0,2}^{2,0}\left[\left.\frac{\gamma_{\mathrm{D}}}{\overline{\gamma_{\mathrm{D}}}} \prod_{i=1}^{2} \frac{m_{i}}{\Omega_{i}}\right|_{m_{1}, m_{2}}\right] G_{1,3}^{2,1}\left[\left.\frac{\gamma_{\mathrm{D}}}{\overline{\gamma_{\mathrm{E}}}} \prod_{j=1}^{2} \frac{m_{j}}{\Omega_{j}}\right|_{m_{1}, m_{2}, 0} ^{1}\right] d \gamma_{\mathrm{D}} \\
& =\frac{1}{\prod_{i=1}^{2} \Gamma\left(m_{i}\right) \prod_{j=1}^{2} \Gamma\left(m_{j}\right)} \times \\
& G_{2,2: 0,2: 1,3}^{2,1: 2,0: 2,1}\left[\begin{array}{c}
0,1 \\
0,0
\end{array}\left|m_{1}, m_{2}\right| m_{1}, m_{2}, 0 \mid \frac{1}{\overline{\gamma_{D}}} \prod_{i=1}^{2} \frac{m_{i}}{\Omega_{i}}, \frac{1}{\overline{\gamma_{E}}} \underset{j=1}{2} \frac{m_{j}}{\Omega_{j}}\right]
\end{aligned}
$$

$M_{2}$ is given as

$$
\begin{aligned}
& M_{2}=\frac{1}{\prod_{i=1}^{2} \Gamma\left(m_{i}\right) \prod_{j=1}^{2} \Gamma\left(m_{j}\right)} \times \\
& G_{2,2: 0,2: 1,3,3}^{2,1: 2,2,1}\left[\begin{array}{c|c|c|c}
0,1 & - & 1 \\
0,0 & m_{1}, m_{2} & m_{1}, m_{2}, 0 & \frac{1}{\overline{\gamma_{\mathrm{E}}}} \prod_{j=1}^{2} \frac{m_{j}}{\Omega_{j}}, \frac{1}{\gamma_{\mathrm{D}}} \prod_{i=1}^{2} \frac{m_{i}}{\Omega_{i}}
\end{array}\right]
\end{aligned}
$$

$M_{3}$ is given as

$$
M_{3}=\frac{1}{\prod_{j=1}^{2} \Gamma\left(m_{j}\right)} G_{2,4}^{4,1}\left[\left.\frac{1}{\overline{\gamma_{\mathrm{E}}}} \prod_{j=1}^{2} \frac{m_{j}}{\Omega_{j}}\right|_{m_{1}, m_{2}, 0,0} ^{0,1}\right]
$$

\section{Numerical Results}

In this section, we present Monte-Carlo simulations to confirm the derived analytical results. 
Figure 2 shows the ASC performance. $\bar{\gamma}=10 \mathrm{~dB}$. Table 1. shows the simulation parameters. From Figure 2., it is found that the analytical results perfectly match with the simulation results. For a fixed $K$, the ASC performance is improved with increasing $m_{\mathrm{D}}$ and decreasing $m_{\mathrm{E}}$. The ASC performance for $(2,1)$ is better than that of $(1,1)$ and $(1,2)$. Further, increasing $K$ improves the ASC performance. This is because a higher $K$ means that the $\mathrm{S} \rightarrow \mathrm{D}$ channel is better than the $\mathrm{S} \rightarrow \mathrm{E}$ channel.

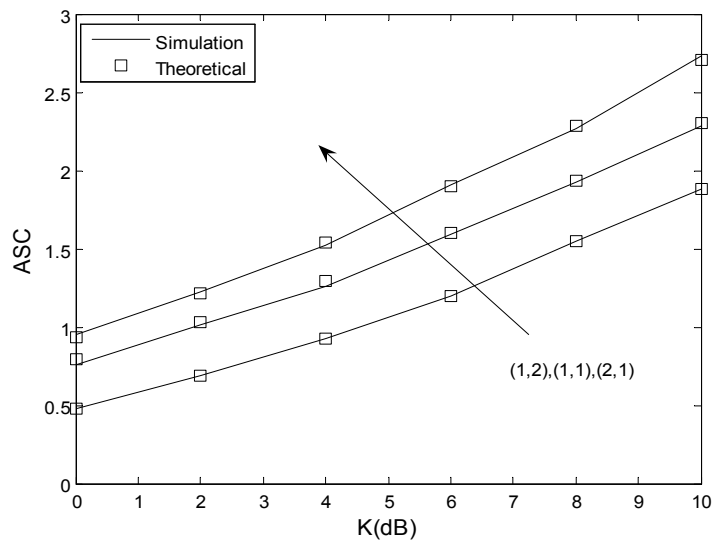

Fig. 2. ASC performance versus $K$.

Table 1. Simulation Parameters

\begin{tabular}{|c|c|c|c|}
\hline$m_{\mathrm{D}}$ & 1 & 1 & 2 \\
\hline$m_{\mathrm{E}}$ & 2 & 1 & 1 \\
\hline$G_{\mathrm{D}}$ & $5 \mathrm{~dB}$ & $5 \mathrm{~dB}$ & $5 \mathrm{~dB}$ \\
\hline $\mathrm{G}_{\mathrm{E}}$ & $5 \mathrm{~dB}$ & $5 \mathrm{~dB}$ & $5 \mathrm{~dB}$ \\
\hline
\end{tabular}

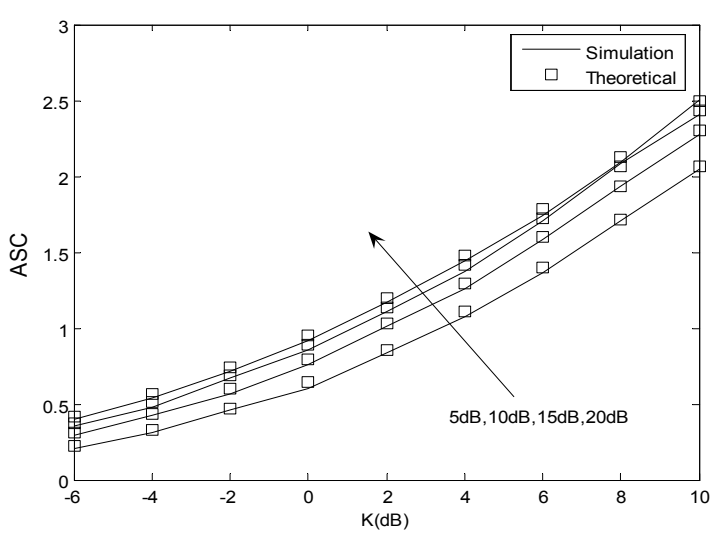

Fig. 3. ASC performance versus $K$. 
Figure 3. presents the ASC performance versus $K$ with $(1,1) . \bar{\gamma}=5 \mathrm{~dB}, 10 \mathrm{~dB}, 15 \mathrm{~dB}, 20$ $\mathrm{dB}, G_{\mathrm{D}}=G_{\mathrm{E}}=5 \mathrm{~dB}, N_{\mathrm{D}}=N_{\mathrm{E}}=2$. It is found that the simulation results perfectly match the analytical results. For fixed $K$, increasing $\bar{\gamma}$ improves the ASC performance. This is because the $\mathrm{S} \rightarrow \mathrm{D}$ channel is better than the $\mathrm{S} \rightarrow \mathrm{E}$ channel.

\section{Conclusions}

In this paper, the ASC performance of the mobile image transmission networks is investigated. We derive exact closed-form ASC expressions. They are verified via MonteCarlo simulations.Increasing $K$ improves the quality of mobile image transmission networks.

Acknowledgments. This project was supported by Shandong Province Natural Science Foundation(no.ZR2017BF023), China Postdoctoral Science Foundation funded project(no.2017M612223),National Natural Science Foundation of China (no. 61402246,61771271), Shandong Province Postdoctoral Innovation Project(no. 201703032).

\section{References}

[1] Chen,S., and Zhao,J., "The requirements, challenges and technologies for 5G of terrestrial mobile telecommunication,'IEEE Communications Magazine, Vol. 52, No. 5, pp. 36-43(2014).

[2] Boccardi,F., Heath, R. W., Lozano,A., Marzetta,T. L., and Popovski,P. "Five disruptive technology directions for 5G,'IEEE Communications Magazine, Vol. 52, No. 2, pp. 74-80(2014).

[3] Maheswari,S. U., Hemanth,D. J."Performance enhanced image steganography systems using transforms and optimization techniques," Multimedia Tools and Applications,Vol. 76, No. 1,pp. 415-436(2017).

[4] Seunghwan, C., Sungju, L.,Yeonwoo, L.,Changsun, K.,Taikyeong, J.,’Secure Video Transmission on Smart Phones for Mobile Intelligent Network," International Journal of Security and its Applications, Vol. 7, No. 1, pp. 143-154(2013).

[5] Zhang ,K., Liang, X., Shen,X., Lu,R."Exploiting multimedia services in mobile social networks from security and privacy perspectives," IEEE Communications Magazine, Vol. 52, No. 3, pp.5865(2014).

[6] Zhang,X.,Mckay,R.M.,Zhou,X.,Heath,R.W.”Artificial-noise-aided secure multi-antenna transmission with limited feedback." IEEE Transactions on Communications, Vol.14,No.5,pp. 2742-2754(2015).

[7] Khandaker,M. R. A., and Wong,K. "Masked beamforming in the presence of energy-harvesting eavesdroppers,"IEEE Transactions on Information Forensics and Security, Vol. 10, No. 1, pp.4054(2015).

[8] Ilhan, H., Uysal, M. and Altunbas, I., "Cooperative Diversity for Intervehicular Communication: Performance Analysis and Optimization," IEEE Transactions on Vehicular Technology, Vol. 58, No.7, pp. 3301-3310 (2009).

[9] Ochiai, H., Mitran, P. and Tarokh, V., "Variable-Rate Two-Phase Collaborative Communication Protocols for Wireless Networks," IEEE Transactions on Vehicular Technology, Vol. 52, No. 9, pp. 4299-4313(2006).

[10] Bloch,M.,Barros, J. ,Rodrigues,M. R., and McLaughlin,S. W. "Wireless information-theoretic security," IEEE Transactions on Information Theory, Vol. 54, no. 6, pp. 2515-2534(2008).

[11] Gradshteyn,I., and Ryzhik, I. Table of Integrals, Series and Products, 7th ed., San Diego,CA, USA: Academic Press, 2007. 